\title{
Chlorophyll $a$ Fluorescence Parameters of Hulled and Hull-less Barley (Hordeum vulgare L.) DH Lines Inoculated with Fusarium culmorum
}

\author{
Tomasz Warzecha (iD ${ }^{1 *}$, Edyta Skrzypek ${ }^{2}$, Tadeusz Adamski ${ }^{3}$, Maria Surma ${ }^{3}$, Zygmunt Kaczmarek ${ }^{3}$, and \\ Agnieszka Sutkowska ${ }^{1}$ \\ ${ }^{1}$ University of Agriculture in Kraków, Department of Plant Breeding and Seed Science Lobzowska 24, 31-140 Kraków, \\ Poland \\ ${ }^{2}$ Polish Academy of Sciences, The Franciszek Górski Institute of Plant Physiology, Niezapominajek 21, 30-239 Kraków, \\ Poland \\ ${ }^{3}$ Institute of Plant Genetics, Polish Academy of Sciences, Strzeszyńska 34, 60-681 Poznań, Poland
}

(Received on July 4, 2018; Revised on October 17, 2018; Accepted on February 12, 2019)

Barley worldwide is affected seriously by Fusarium seedling blight (FSB) and Fusarium head blight (FHB) diseases caused by the Fusarium species. The objective of this study was to facilitate the resistance of hulled and hull-less barley at different growth stages to $F$. culmorum according to direct parameters: disease rating (DR), fresh weight of leaves and roots, kernel weight per spike, kernel number per spike, plump kernels, and indirect parameters - chlorophyll $a$ fluorescence (CF). Plate assay, greenhouse and field tests were performed on 30 spring barley doubled haploid (DH) lines and their parents infected with Fusarium culmorum. Direct parameters proved that hulled genotypes show less symptoms. Most studied chlorophyll $a$ fluorescence (CF) parameters (apart from DIo/CS - amount of energy dissipated from PSII for laboratory test, TRo/CS - amount of excitation energy trapped in PSII reaction centers, ETo/CS - amount of energy used for electron transport and $\mathrm{RC} / \mathrm{CS}$ - number of active reaction centres in the state of fully reduced PSII reaction center in field experiment) were significantly affected by $F$.

*Corresponding author.

Phone) +48-12-6333606 ext.111, FAX) +48-12-6333606 ext.129

E-mail) rrwarzec@cyf-kr.edu.pl

ORCID

Tomasz Warzecha

http://orcid.org/0000-0002-7121-0123

(c) This is an Open Access article distributed under the terms of the Creative Commons Attribution Non-Commercial License (http:// creativecommons.org/licenses/by-nc/4.0) which permits unrestricted noncommercial use, distribution, and reproduction in any medium, provided the original work is properly cited.

Articles can be freely viewed online at www.ppjonline.org. culmorum infection. In all experiments, hulled genotypes had higher values of CF parameters compared to hull-less ones. Significant correlations were detected between direct and indirect parameters and also between various environments. It was revealed that $\mathrm{ABS} / \mathrm{CS}$, TRo/CS, and RC/CS have significant positive correlation in greenhouse test and field experiment. Significant correlations suggest the possibility of applying the CF parameters in selection of barley DH lines resistant to $F$. culmorum infection.

Keywords : chlorophyll $a$ fluorescence, fungal infection, Fusarium head blight, Fusarium seedling blight, spring barley

Handling Editor : Jeon, Junhyun

Fusarium seedling blight (FSB) and Fusarium head blight (FHB) are devastating diseases caused by the Fusarium species in barely worldwide (Inch and Gilbert, 2003; Magan et al., 2002; Marin et al., 2013; Warzecha et al., 2011). The recent infection of seedlings in temperate EU conditions is mainly caused by Microdochium spp, which are also heavily implicated in FHB (Nielsen et al., 2014). Studies of European commercial barley crops have indicated that the FHB complex reported on the crop is much more diverse than previously $F$. graminearum ( $F$. graminearum sensu stricto), $F$. culmorum and $F$. poae, mixed populations of newly emerging $F$. langsethiae, $F$. avenaceum, F. tricinctum and Microdochium nivale and Microdochium majus (Nielsen et al., 2011, 2013).

Moreover, F. culmorum is considered most aggressive 
to wheat seedlings along with $M$. nivale as reported by Imathiu et al. (2009). The diseases caused the reduction of grain yield and quality as a result of contamination with mycotoxins, which are responsible for mycotoxicoses in humans and domestic animals (Arseniuk et al., 1999; Bottalico and Perrone, 2002; Buerstmayr et al., 2009; Mardi et al., 2006; Marin et al., 2013; Wiśniewska et al., 2014). The malting and brewing industry also suffers because of contaminated grain (Desjardins, 2006; Ma et al., 2009).

Two components of resistance to FHB have been postulated - resistance to initial infection (type I) and resistance to infection spread (type II) (Schroeder and Christensen, 1963). Mesterházy (1995) and Mesterházy et al. (1999) proposed additional components such as resistance to kernel infection (type III) and resistance to toxin accumulation (type V) (Foroud and Eudes, 2009). When considering resistance to FHB, the reduction of yield and/or yield-related traits after artificial inoculation have been accepted as the indirect measures of toxin degradation - resistance type IV (Buerstmayr et al., 2009; Chełkowski et al., 2000; Warzecha et al., 2010). As a direct assessment, visual scoring of disease symptoms on spikes is utilized to determine FHB severity (Buerstmayr et al., 2009; Yang et al., 2005). The severity of FHB is a quantitative trait modulated particularly temperature and rainfall, from flowering to the soft-dough-stage of kernel development (Adamski et al. 1999). Therefore, selection of barley genotypes with improved resistance to FHB is difficult (Chełkowski et al., 2000; Cowger et al., 2009; Mesterházy, 1995, 2002; Miedaner, 1997; Snijders, 2004). Also, significant genotype-environment interaction could be observed (Buerstmayr et al., 2009; Miedaner et al., 2001; Warzecha et al., 2011).

The photosynthetic performance of plants may provide useful information about their abilities to fight biotic stress caused by infection. Therefore, insights into plant productivity can be investigated by studying the activity of the photosynthetic apparatus such as chlorophyll fluorescence (CF) kinetics (Bolhar-Nordenkampf and Öquist, 1993). Such studies provide crucial information about the photosystem II (PSII) and its photochemical efficiency as well as give information about the amount of excitation energy trapped in PSII reaction centres during photosynthesis. Additionally, CF measurements give the opportunity to assess light energy absorption, amount of energy dissipated from PSII, numbers of active reaction centres oxidized and reduced, and energy quantum used for electron transport (Baker and Rosenqvist, 2004). Additional clarification of the molecular function of $\mathrm{CF}$ and their relations with assimilation pigments might have potential for yield enhancement, as was reported by Fracheboud and Leipner (2003),
Maxwell and Johnson (2000), O’Neill et al. (2006), Pereira et al. (2000), and Smillie and Nott (1982). Fluorescence parameters have been used to detect even slight differences in activity of the photosynthetic apparatus among genotypes of crop plants. There are several reports on the CF imaging (CFI) technique application to detect changes in photosynthesis induced by pathogen (Gorbe and Calatayud, 2012; Rolfe and Scholes, 2010), or to detect Fusarium head blight caused by $F$. culmorum on wheat heads artificially inoculated (Bauriegel et al., 2010). The use of CFI technique is limited due to its high cost and inconvenient equipment, additional problems are as follows: difficulties in dark adopting and applying even illumination, time requirements for imaging large number of plants in consequence limitation of biological replication (Ajigboye et al., 2016). Pulse modulated amplitude (PAM), including CFI, is used in most studies (Ajigboye et al., 2016). The alternative, non-invasive, sensitive and rapid technique is the fast induced fluorescent rise technique termed OJIP (Transient chlorophyll $a$ fluorescence rise induced during a dark to strong light transition), in reference to the sequence of transients reported upon the rise of fluorescence from darkness induced by light. OJIP is sensitive to changes in PSII effectiveness similar to fluorescence measurement (Ajigboye et al., 2014, 2016; Demetriou et al., 2007; Živcák et al., 2008). So far use in detecting and quantifing of the effect of fungal pathogen infection has not been widely explored. There is a report describing studies undertaken to identify chlorophyll fluorescence (CF) parameters that can quantify changes in PSII linked with plant responses in three different wheat pathosystems of foliar, stem-base and ear diseases (Ajigboye et al., 2016).

There are no literature data providing complex characteristics of chlorophyll fluorescence parameters such as light energy absorption (ABS/CS), amount of excitation energy trapped in PSII reaction centers (TRo/CS), amount of energy used for electron transport (ETo/CS), amount of energy dissipated from PSII (DIo/CS), equal to [ABS/CS - TRo/ $\mathrm{CS}]$ ), number of active reaction centres in the state of fully oxidized PSII reaction center (RC/CSo), number of active reaction centres in the state of fully reduced PSII reaction center $(\mathrm{RC} / \mathrm{CS})$ in completely homozygous material such as barley DH lines, following Fusarium culmorum infection, performed in different environments and growth stages. Although, identifying physiological markers associated with resistance to FSB and FHB are especially compelling for eventual use in selection. Additionally, there are numerous publications describing evaluation of FHB resistance and the associated QTLs in wheat but very few studies on FSB resistance or both FHB and FSB observed in the same 
material (Ren et al., 2015).

Therefore the aims of this study were: (1) to determine the susceptibility of hulled and hull-less barley DH lines to Fusarium culmorum at a young growth stage with two methods of infection: in laboratory test (plate assay for resistance with mycelium as infectious agent) - environment 1 , in greenhouse test (with spores as infectious agent) - environment 2, and at adult growth stage in field test environment 3, (2) to assess the impact of head infection on chlorophyll $a$ fluorescence of the flag leaf as a main photosynthetic organ. All genotypes underwent direct assessment of disease rating (DR), reduction of fresh weight, as well as reduction of yield-related traits and indirect assessment by using selected chlorophyll $a$ fluorescence parameters (CF). To facilitate more resistant $\mathrm{DH}$ lines, disease rating and fresh weight of seedlings were assigned with chlorophyll $a$ fluorescence parameters. In order to test the above associations, correlation coefficients were calculated.

\section{Materials and Methods}

Plant material. Material for the study included 32 spring barley (Hordeum vulgare L.) lines: 2 parental genotypes (hull-less line 1N86 and hulled line RK63/1), and 30 doubled haploid (DH) lines derived from $\mathrm{F}_{1}$ hybrids RK63/1 $\times 1 \mathrm{~N} 86$. The parental lines differ in susceptibility to $F u$ sarium culmorum infection. Line 1N86 was considered susceptible and RK63/1 resistant (Warzecha et al., 2015). $\mathrm{DH}$ lines generated from the crossing of $1 \mathrm{~N} 86$ and RK63/1 were developed by the Hordeum bulbosum technique. Standard procedures were applied for crossing $H$. vulgare with $H$. bulbosum and in vitro culture of immature embryos (Kasha and Kao, 1970; Pickering and Devaux, 1992).

Plate assay. Inoculation of semi-germinated seeds was performed with an isolate of Fusarium culmorum (Plant Breeding Institute, Wageningen, Holland) culture in Petri dishes on a PDA medium (Potatoe Dextrose Agar Sigma) at a temperature of $22^{\circ} \mathrm{C}$ without access to light, in a microbiological incubator (B 6060 Heraeus, USA) over a period of seven days. The kernels were surface disinfected for 15 min with $20 \%$ Domestos solution (commercial bleach, with sodium hypochlorite as the active ingredient) and substantially washed three times in sterile water and placed on blotting paper for $24 \mathrm{~h}$ for germination. Then the semi-germinated seeds were transferred onto PDA medium discs (ø $4 \mathrm{~mm}$ ) overgrown with Fusarium culmorum mycelium. Barley kernels placed on sterile medium discs were the control. The test was repeated three times in February 2013. In each test 20 grains of each genotype were sown on Petri dishes for control and inoculated combinations. Therefore, the replication was a set of 20 plants of the same genotype. The assay for resistance was carried out in an airconditioned chamber over a period of 7 days at $22^{\circ} \mathrm{C} / 20^{\circ} \mathrm{C}$ (day/night), with $130 \mu \mathrm{E} / \mathrm{m}^{2} / \mathrm{s}$ lighting, $12 / 12 \mathrm{~h}$ photoperiod and $100 \%$ relative humidity. To determine the effect of infection on seedling development, direct assessment using a disease rating (DR) (Chełkowski and Mańka, 1983) was calculated according to the formula:

$$
\mathrm{DR} \%=100 \times\left(\mathrm{n}_{\mathrm{i}} \mathrm{D}_{\mathrm{i}}\right) / \mathrm{ND}_{\text {max }}
$$

where $\mathrm{n}_{\mathrm{i}}-$ the number of plants of the $i$ th category, $i$ th category - certain value from the $0-5$ scale assessed by visual symptoms of disease where 0 - no symptoms, 5 complete damage (over $80 \%$ of organ is damaged)

$\mathrm{D}_{\mathrm{i}}$ - numerical value of the $i$ th category (ranged between $0-5$,

$\mathrm{N}$ - the total number of plants in the sample, $\mathrm{D}_{\max }-$ is the maximum value in $0-5$ disease scale, Wojciechowski et al. (1997).

Chlorophyll fluorescence parameters were measured on the fully developed leaves of 7-day seedlings. The fresh weight (FW, mg) of leaves and roots of inoculated and control seedlings was also determined in each replication.

Production of inoculums for greenhouse and field test. The $300 \mathrm{ml}$ Erlenmeyer flasks were filled with $50 \mathrm{~g}$ of wheat grain and $15 \mathrm{ml}$ of water (40\% moisture). After 24 $\mathrm{h}$, they were autoclaved for $30 \mathrm{~min}$ at $1 \mathrm{~atm}$. at a temperature of $121^{\circ} \mathrm{C}$ and then cooled. Fusarium culmorum isolate derived from SNA (Synthetic Nutrient Agar medium) medium (Chełkowski and Mańka, 1983) were transferred into the Erlenmeyer flasks $(250 \mathrm{ml})$.

The methods of infection. After transferring on SNA slants, mycelium grew in 2-3 days, then was eluted with 1-2 $\mathrm{ml}$ of sterile water, mixed, scraped from the surface of the SNA slant and poured on the grain surface.

After inoculation, the flasks were incubated at a temperature of $22^{\circ} \mathrm{C}$ without access to light, in a microbiological incubator (B 6060 Heraeus, USA) and every second day were shaken in order to infect all grains uniformly. Inoculum production lasted approximately 3-4 weeks.

Preparation of inoculum to infect kernels. The spores were eluted with sterile water and filtered through several layers of gauze. Spore concentration was determined in a Neubauer chamber. The spore suspension was appropriately diluted to a concentration of $5 \times 10^{6}$ spores $/ \mathrm{ml}$. 


\section{Greenhouse test}

Inoculation of kernels. Grains were soaked in a 5\% solution of carboxymethylcellulose as a superadsorbent agent it adheres to the grains by dipping or coating. Grains were dried on Petri dish, and afterwards obtained a dry gel coating. After drying, coated grains were soaked for $1 \mathrm{~h}$ in a spore suspension $-10 \mathrm{ml}$ per plate and shaken every five minutes. Control grains were soaked in sterile water.

Grains were dried at room temperature for $12 \mathrm{~h}$ and then sown in pots with sterile soil (Arseniuk et al., 1993).

The greenhouse assay for resistance was carried out at $18 / 13^{\circ} \mathrm{C}$ day/night, a photoperiod of $16 / 8 \mathrm{~h}$ light/darkness, and a light intensity $350 \mu \mathrm{E} / \mathrm{m}^{2} / \mathrm{s}$.

In each combination (inoculated and control) 20 grains of each genotype were sown in sterile soil in three replications (altogether sixty grains of each line). One replication was a set of 20 plants of the same genotype.

Chlorophyll fluorescence was measured on the fully developed leaves of 14-day seedlings. After two weeks the plants were removed from the soil and necrosis or leaf discoloration, fresh weight of leaves (FW) and DR of leaves were determined in control and inoculated plants.

To determine the effect of infection on plant development under greenhouse conditions, the disease rating was calculated according to the formula given above, as described for plate assay resistance.

Field test. Field experiments were carried out in Prusy, South Poland $\left(20^{\circ} 05^{\prime} \mathrm{E} 50^{\circ} 05^{\prime} \mathrm{N}\right)$. The average weather conditions (temperature and rain fall) during the vegetation season was as follows: March $-0.9^{\circ} \mathrm{C}, 32.3 \mathrm{~mm}$; April $8.8^{\circ} \mathrm{C}, 20.1 \mathrm{~mm}$; May $14.2^{\circ} \mathrm{C}, 98.8 \mathrm{~mm}$; June $17.6^{\circ} \mathrm{C}$, $213.1 \mathrm{~mm}$; July $19.2^{\circ} \mathrm{C}, 27.2 \mathrm{~mm}$. The weather records were obtained from the weather station next to the experimental plots. The experiment with 32 genotypes and 2 treatments (inoculation and control) was carried out in a randomized block design. The experimental area was divided into 2 large plots (LP, one for control and one for inoculation. Each LP was divided into three blocks, one block was one replication and genotypes were randomly distributed inside each block. In each plot inside the block, seeds were sown in six 2-m rows, $20 \mathrm{~cm}$ apart, and 200 seeds in each row.

Each line was artificially inoculated with $F$. culmorum. At full anthesis (Zhadoks scale 67), 40 spikes of each line in each replication were sprayed (each spike separately) with $2 \mathrm{ml}$ of conidial suspension $\left(5 \times 10^{6}\right.$ in $\left.1 \mathrm{ml}\right)$ of $F$. culmorum (W.G.Sm) Sacc., isolate KF350 (IPO348-01, ITEM6249) (Warzecha et al., 2010).

Chlorophyll $a$ fluorescence parameters were measured on flag leaves two weeks after inoculation. DR of heads, kernel weight per spike (KWS), kernel number per spike (KNS) and kernel fractions: $>2.5 \mathrm{~mm}$ (KD1). 2.5-2.2 mm (KD2) and $<2.2 \mathrm{~mm}(\mathrm{KD} 3)$, in \%) were examined in control and inoculated plants.

Chlorophyll $a$ fluorescence parameters. Chlorophyll $a$ fluorescence parameters were measured using a fluorometer (Handy PEA; Hansatech Instruments, King's Lynn, Norfolk, UK) at $24^{\circ} \mathrm{C}$ after $20 \mathrm{~min}$ for the detached leaves to adapt to the dark conditions. The measurements were done using a saturating pulse of $3000 \mu \mathrm{mol} / \mathrm{m}^{2} / \mathrm{s}$, pulse duration of $1 \mathrm{~s}$, and fixed gain $(1.0 \times)$. Leaves of three plants of each line were measured in each block. The fluorescence intensity is measured and expressed in relative units. The Kautsky curve or chlorophyll fluorescence transient describes the fluorescence response change over time (Force et al., 2003). Using input data from the fluorescence transient, a group of fluorescence parameters, called the JIPtest, that quantify the stepwise flow of energy through Photosystem II was formulated by Strasser and Strasser (1995).

The following parameters were calculated: light energy absorption (ABS/CS), amount of excitation energy trapped in PSII reaction centers (TRo/CS), amount of energy used for electron transport (ETo/CS), amount of energy dissipated from PSII (DIo/CS), equal to $[\mathrm{ABS} / \mathrm{CS}-\mathrm{TRo} /$ $\mathrm{CS}]$ ), number of active reaction centres in the state of fully oxidized PSII reaction center ( $\mathrm{RC} / \mathrm{CS}$ ), number of active reaction centres in the state of fully reduced PSII reaction center (RC/CS) (Czyczyło-Mysza et al., 2013; Strasser and Tsimillini-Michael, 1998; Strasser et al., 2000).

Statistical analysis. For the examined parameters, a twoway analysis of variance using the independent system was conducted. The distinguished sources of variability were tested using the fixed model. The evaluation of the correlations between characteristics was performed on the basis of the Pearson linear correlation coefficient. Statistical analysis was performed with the application of Statistica StatSoft, Inc.

\section{Results}

The impact of $F$. culmorum infection was significant for most of the studied chlorophyll fluorescence (CF) parameters (apart from DIo/CS for laboratory test, ETo/CS and $\mathrm{RC} / \mathrm{CSo}$ in field experiment) (Tables 1, 2). A significant influence of genotype on chlorophyll fluorescence parameters in all three experiments (plate assay, greenhouse and field tests) was also recorded. Treatment $\times$ genotype inter- 
Table 1. Mean values $\pm \mathrm{SE}$ of chlorophyll $a$ fluorescence $(\mathrm{CF})$ parameters and direct parameters (DR - disease rating, $\mathrm{FW}-$ fresh weigh of leaves [mg] and yield-related traits) of inoculated and control barley DH lines measured at different tests

\begin{tabular}{|c|c|c|c|c|c|c|}
\hline \multirow{2}{*}{$\begin{array}{l}\text { Parameter, } \\
\text { trait }\end{array}$} & \multicolumn{2}{|c|}{ DH lines } & \multicolumn{2}{|c|}{ Parent - RK63/1 } & \multicolumn{2}{|c|}{ Parent - 1N86 } \\
\hline & Inoculated & Control & Inoculated & Control & Inoculated & Control \\
\hline \multicolumn{7}{|c|}{ Plate assay } \\
\hline $\mathrm{ABS} / \mathrm{CS}$ & $309.9 \pm 2.26$ & $316.0 \pm 1.66$ & $310.7 \pm 2.71$ & $312.9 \pm 3.27$ & $316.4 \pm 5.46$ & $306.2 \pm 8.73$ \\
\hline $\mathrm{TR}_{\mathrm{O}} / \mathrm{CS}$ & $251.2 \pm 1.81$ & $256.8 \pm 1.22$ & $256.3 \pm 2.24$ & $256.1 \pm 2.63$ & $256.2 \pm 4.10$ & $250.8 \pm 5.95$ \\
\hline $\mathrm{ET}_{\mathrm{O}} / \mathrm{CS}$ & $111.3 \pm 1.53$ & $117.2 \pm 1.09$ & $130.1 \pm 2.69$ & $118.0 \pm 1.74$ & $114.5 \pm 2.14$ & $117.9 \pm 1.84$ \\
\hline $\mathrm{DI}_{\mathrm{O}} / \mathrm{CS}$ & $58.8 \pm 0.63$ & $59.2 \pm 0.61$ & $54.4 \pm 0.91$ & $56.8 \pm 0.65$ & $60.2 \pm 1.95$ & $55.4 \pm 2.78$ \\
\hline $\mathrm{RC} / \mathrm{CSo}$ & $108.1 \pm 1.15$ & $117.4 \pm 0.80$ & $118.4 \pm 1.64$ & $118.3 \pm 0.97$ & $112.7 \pm 2.33$ & $118.9 \pm 1.53$ \\
\hline $\mathrm{RC} / \mathrm{CS}$ & $578.2 \pm 7.31$ & $634.1 \pm 5.32$ & $676.9 \pm 3.27$ & $652.9 \pm 5.79$ & $598.8 \pm 15.31$ & $660.3 \pm 5.70$ \\
\hline DR - leaf & $23.9 \pm 2.09$ & $0.0 \pm 0.0$ & $7.0 \pm 0.94$ & $0.0 \pm 0.0$ & $58.3 \pm 5.21$ & $0.0 \pm 0.0$ \\
\hline DR - root & $67.03 \pm 1.28$ & $3.3 \pm 0.49$ & $58.7 \pm 9.25$ & $6.3 \pm 0.82$ & $89.8 \pm 3.25$ & $8.6 \pm 0.54$ \\
\hline FW - leaf & $64.1 \pm 2.10$ & $85.3 \pm 2.11$ & $70.0 \pm 1.52$ & $80.0 \pm 1.32$ & $56.7 \pm 4.74$ & $90.0 \pm 3.28$ \\
\hline FW - root & $24.6 \pm 1.20$ & $67.7 \pm 3.20$ & $33.3 \pm 5.67$ & $90.0 \pm 6.12$ & $16.7 \pm 2.58$ & $56.7 \pm 4.26$ \\
\hline \multicolumn{7}{|c|}{ Greenhouse test } \\
\hline $\mathrm{ABS} / \mathrm{CS}$ & $270.9 \pm 5.00$ & $335.6 \pm 1.21$ & $287.4 \pm 5.75$ & $333.6 \pm 11.92$ & $249.4 \pm 14.34$ & $331.0 \pm 10.40$ \\
\hline $\mathrm{TR}_{\mathrm{O}} / \mathrm{CS}$ & $217.8 \pm 4.07$ & $274.6 \pm 0.97$ & $233.9 \pm 4.40$ & $273.2 \pm 9.80$ & $199.1 \pm 12.04$ & $270.4 \pm 8.70$ \\
\hline $\mathrm{ET}_{\mathrm{O}} / \mathrm{CS}$ & $107.7 \pm 2.54$ & $146.0 \pm 0.76$ & $117.4 \pm 4.45$ & $145.3 \pm 6.27$ & $97.7 \pm 7.17$ & $143.2 \pm 6.19$ \\
\hline $\mathrm{DI}_{\mathrm{O}} / \mathrm{CS}$ & $53.4 \pm 1.19$ & $61.0 \pm 0.29$ & $53.5 \pm 1.35$ & $60.4 \pm 2.22$ & $50.3 \pm 3.73$ & $60.6 \pm 1.72$ \\
\hline RC/CSo & $96.7 \pm 2.24$ & $131.6 \pm 0.82$ & $102.5 \pm 5.35$ & $129.6 \pm 6.21$ & $84.3 \pm 6.92$ & $131.5 \pm 8.44$ \\
\hline $\mathrm{RC} / \mathrm{CS}$ & $501.4 \pm 12.78$ & $725.5 \pm 4.86$ & $551.0 \pm 26.12$ & $716.0 \pm 35.03$ & $421.8 \pm 46.21$ & $719.1 \pm 48.43$ \\
\hline DR - leaf & $58.1 \pm 2.46$ & $3.3 \pm 1.15$ & $33.1 \pm 4.68$ & $6.3 \pm 2.08$ & $80.7 \pm 5.20$ & $8.6 \pm 4.03$ \\
\hline FW - leaf & $139.0 \pm 4.78$ & $246.7 \pm 3.89$ & $126.7 \pm 5.02$ & $203.3 \pm 8.26$ & $60.0 \pm 3.12$ & $183.3 \pm 10.30$ \\
\hline \multicolumn{7}{|c|}{ Field test } \\
\hline $\mathrm{ABS} / \mathrm{CS}$ & $599.3 \pm 6.65$ & $666.6 \pm 9.51$ & $622.3 \pm 66.67$ & $704.3 \pm 12.69$ & $625.5 \pm 34.82$ & $658.2 \pm 9.58$ \\
\hline $\mathrm{TR}_{\mathrm{O}} / \mathrm{CS}$ & $470.6 \pm 5.47$ & $499.3 \pm 7.19$ & $485.0 \pm 69.31$ & $534.5 \pm 4.95$ & $502.0 \pm 22.01$ & $523.2 \pm 5.98$ \\
\hline $\mathrm{ET}_{\mathrm{O}} / \mathrm{CS}$ & $194.9 \pm 4.05$ & $191.2 \pm 4.65$ & $209.2 \pm 43.10$ & $206.7 \pm 15.16$ & $232.6 \pm 11.12$ & $215.4 \pm 5.00$ \\
\hline $\mathrm{DI}_{\mathrm{O}} / \mathrm{CS}$ & $128.8 \pm 2.90$ & $167.3 \pm 7.46$ & $137.2 \pm 23.77$ & $169.8 \pm 8.65$ & $123.5 \pm 15.06$ & $135.0 \pm 4.28$ \\
\hline $\mathrm{RC} / \mathrm{CSo}$ & $168.2 \pm 2.77$ & $171.7 \pm 3.04$ & $171.1 \pm 29.84$ & $177.0 \pm 10.04$ & $196.0 \pm 6.51$ & $180.7 \pm 4.96$ \\
\hline $\mathrm{RC} / \mathrm{CS}$ & $845.3 \pm 19.66$ & $781.8 \pm 24.39$ & $839.7 \pm 202.6$ & $808.5 \pm 93.25$ & $1022.1 \pm 95.26$ & $900.6 \pm 34.74$ \\
\hline DR - head & $61.3 \pm 3.24$ & - & $48.2 \pm 2.98$ & - & $64.0 \pm 5.12$ & - \\
\hline KNS & $14.4 \pm 2.95$ & $19.8 \pm 2.01$ & $16.2 \pm 1.96$ & $19.6 \pm 1.02$ & $21.5 \pm 1.15$ & $23.9 \pm 0.98$ \\
\hline KWS & $0.43 \pm 0.12$ & $0.88 \pm 0.10$ & $0.48 \pm 0.12$ & $0.98 \pm 0.13$ & $0.44 \pm 0.16$ & $0.95 \pm 0.09$ \\
\hline KD1 & $28.2 \pm 12.57$ & $72.6 \pm 9.24$ & $28.6 \pm 10.23$ & $60.3 \pm 11.25$ & $49.1 \pm 14.2$ & $74.4 \pm 12.8$ \\
\hline $\mathrm{KD} 2$ & $34.5 \pm 9.77$ & $18.9 \pm 7.56$ & $39.3 \pm 5.23$ & $33.0 \pm 6.31$ & $36.9 \pm 7.12$ & $18.4 \pm 2.35$ \\
\hline KD3 & $37.3 \pm 12.14$ & $8.6 \pm 2.41$ & $32.0 \pm 6.32$ & $6.6 \pm 1.21$ & $14.0 \pm 2.51$ & $7.2 \pm 1.12$ \\
\hline
\end{tabular}

KWS - kernel weight per spike, KNS - number of kernels per spike, KD1, KD2 and KD3 - kernel diameter: $>2.5 \mathrm{~mm} .2 .5-2.2 \mathrm{~mm}$ and $<2.2$ $\mathrm{mm}$, respectively

action was important mainly for CF parameters measured in the plate assay and greenhouse test, whereas for the field experiment this interaction was significant (at $P<0.05$ ) only for Dlo/CS. In Supplementary Table 1-3 the mean values of $\mathrm{CF}$ are given for individual lines in control and infected plants. The impact of $F$. culomurm infection was also observed on direct parameters in greenhouse and field tests (Table 2). Infection significantly influenced the examined traits under greenhouse (DR and FW) and field (KNS,
KWS, KD1, KD2, KD3) conditions (Table 2, Supplementary Table 2,4). A significant impact of genotype on studied traits (except DR in field test) was also observed, while interaction between treatment and genotype was significant only for KD3 (Table 2).

In all experiments hulled genotypes had higher values of all measured CF parameters compared to hull-less ones (Supplementary Table 1-3). These data correspond with direct parameters, where hulled genotypes showed less 
Table 2. F-statistic from analysis of variance for chlorophyll $a$ fluorescence $(\mathrm{CF})$ parameters and direct parameters (DR - disease rating, FW - fresh weigh) of inoculated and control barley DH lines measured at different tests

\begin{tabular}{|c|c|c|c|c|c|c|c|c|c|}
\hline \multirow[b]{2}{*}{$\begin{array}{l}\text { CF p } \\
\text { arameters }\end{array}$} & \multicolumn{3}{|c|}{ Plate assay } & \multicolumn{3}{|c|}{ Greenhouse test } & \multicolumn{3}{|c|}{ Field test } \\
\hline & $\begin{array}{c}\mathrm{T} \\
(\mathrm{df}=1)\end{array}$ & $\underset{(d f=31)}{G}$ & $\begin{array}{c}\mathrm{G} \times \mathrm{T} \\
(\mathrm{df}=31)\end{array}$ & $\begin{array}{c}\mathrm{T} \\
(\mathrm{df}=1)\end{array}$ & $\begin{array}{c}\mathrm{G} \\
(\mathrm{df}=31)\end{array}$ & $\begin{array}{c}\mathrm{G} \times \mathrm{T} \\
(\mathrm{df}=31)\end{array}$ & $\begin{array}{c}\mathrm{T} \\
(\mathrm{df}=1)\end{array}$ & $\begin{array}{c}G \\
(\mathrm{df}=31)\end{array}$ & $\begin{array}{c}\mathrm{G} \times \mathrm{T} \\
(\mathrm{df}=31)\end{array}$ \\
\hline $\mathrm{ABS} / \mathrm{CS}$ & $7.39^{* *}$ & $3.34 * *$ & $2.36^{* *}$ & $238.34 * *$ & $2.69^{* *}$ & $2.42 * *$ & $46.36^{* *}$ & $3.13^{* *}$ & 1.19 \\
\hline $\mathrm{TR}_{\mathrm{O}} / \mathrm{CS}$ & $11.09 * *$ & $3.53 * *$ & $2.55 * *$ & $283.61 * *$ & $2.73 * *$ & $2.56^{* *}$ & $13.86^{* *}$ & $3.50 * *$ & 0.77 \\
\hline $\mathrm{ET}_{\mathrm{O}} / \mathrm{CS}$ & $26.12 * *$ & $7.97 * *$ & $3.81 * *$ & $310.69 * *$ & $2.44 * *$ & $2.59 * *$ & 0.31 & $3.21 * *$ & 0.89 \\
\hline $\mathrm{DI}_{\mathrm{O}} / \mathrm{CS}$ & 0.29 & $3.25 * *$ & $1.71^{*}$ & $43.67 * *$ & $1.69^{*}$ & 1.24 & $27.99 * *$ & $1.71 *$ & $1.57^{*}$ \\
\hline $\mathrm{RC} / \mathrm{CSo}$ & $92.54 * *$ & $5.38^{* *}$ & $3.43 * *$ & $292.98 * *$ & $2.15^{* *}$ & $2.12 * *$ & 1.01 & $3.58 * *$ & 0.85 \\
\hline $\mathrm{RC} / \mathrm{CS}$ & $85.37 * *$ & $6.12 * *$ & $3.44 * *$ & $401.41 * *$ & $2.37 * *$ & $2.67 * *$ & $5.20 *$ & $2.62 * *$ & 0.99 \\
\hline DR - leaf & - & $24.35^{* *}$ & - & $355.12 * *$ & $15.31 * *$ & $17.03 * *$ & - & - & - \\
\hline FW - leaf & $51.83^{* *}$ & $1.73 *$ & 0.62 & $201.42 * *$ & $3.91 * *$ & $1.70 *$ & - & - & - \\
\hline DR - Root & $7148.52 * *$ & $7.02 * *$ & $9.11 * *$ & - & - & - & - & - & - \\
\hline FW - Root & $233.51 * *$ & $3.67 * *$ & 1.05 & - & - & - & - & - & - \\
\hline DR - head & - & - & - & - & - & - & & 0.92 & \\
\hline KNS & - & - & - & - & - & - & $101.34 * *$ & $2.51 * *$ & 0.95 \\
\hline KWS & - & - & - & - & - & - & $408.84 * *$ & $2.83 * *$ & 0.91 \\
\hline KD1 & - & - & - & - & - & - & $404.22 * *$ & $4.91 * *$ & 1.11 \\
\hline KD2 & - & - & - & - & - & - & $82.31 * *$ & $2.40 * *$ & 0.91 \\
\hline KD3 & - & - & - & - & - & - & $182.52 * *$ & $3.31 * *$ & $14.64 * *$ \\
\hline
\end{tabular}

$* P<0.05 ; * * P<0.01$

$\mathrm{T}$ - treatment, G - genotype, T x G - treatment x genotype interactionKWS - kernel weight per spike, KNS - number of kernels per spike, $\mathrm{KD} 1, \mathrm{KD} 2$ and $\mathrm{KD} 3$ - kernel diameter: $>2.5 \mathrm{~mm}$. 2.5-2.2 $\mathrm{mm}$ and $<2.2 \mathrm{~mm}$, respectively

Table 3. Orthogonal contrasts for chlorophyll $a$ fluorescence (CF) parameters between hulled and hull-less barley DH lines at plate assay, greenhouse and field tests

\begin{tabular}{|c|c|c|c|c|c|c|}
\hline \multirow{2}{*}{$\mathrm{CF}$ parameters } & \multicolumn{2}{|c|}{ Plate assay } & \multicolumn{2}{|c|}{ Greenhouse test } & \multicolumn{2}{|c|}{ Field test } \\
\hline & Inoculated & Control & Inoculated & Control & Inoculated & Control \\
\hline $\mathrm{ABS} / \mathrm{CS}$ & $16.91 * *$ & $-6.71^{*}$ & $12.31 *$ & 5.10 & $40.80 * *$ & 22.12 \\
\hline $\mathrm{TR}_{\mathrm{O}} / \mathrm{CS}$ & $15.64 * *$ & -4.50 & $12.06^{*}$ & 3.47 & 20.35 & 5.23 \\
\hline $\mathrm{ET}_{\mathrm{O}} / \mathrm{CS}$ & $13.89 * *$ & -2.99 & $9.38 * *$ & 1.53 & 10.12 & -3.77 \\
\hline $\mathrm{DI}_{\mathrm{O}} / \mathrm{CS}$ & 1.27 & $-2.21^{*}$ & -0.88 & 1.68 & $20.42 *$ & 16.89 \\
\hline $\mathrm{RC} / \mathrm{CSo}$ & $11.07 * *$ & $-4.08 * *$ & 4.80 & 4.77 & 6.74 & -4.52 \\
\hline $\mathrm{RC} / \mathrm{CS}$ & $68.54 * *$ & $-17.54 *$ & $44.12 * *$ & 17.36 & 6.73 & -23.18 \\
\hline
\end{tabular}

$* P<0.05 ; * * P<0.01$

disease symptoms. Estimates of differences between hulled and hull-less lines showed that infected hulled lines have had significantly (at $P<0.05$ ) higher values of CF parameters in the plate assay (except Dlo/CS) and the greenhouse test (with an exception for Dlo/CS and $\mathrm{RC} / \mathrm{CSo}$ ), while in control plants these differences were not significant in greenhouse and field tests (Table 3, Supplementary Table 1).

The relationship observed between CF parameters and DR and FW of leaves and roots in the plate assay are presented in Table 4. In most cases (apart from DIo/CS) CF parameters had a significant negative correlation with DR of leaves and roots, which indicates that CF parameters de- creased after inoculation. Higher values of DR resulted in lower performance of the PSII photosystem as its components are affected by infection. In contrast, the fresh weight of leaves had significant positive correlation with ETo/CS, $\mathrm{RC} / \mathrm{CS}$ and $\mathrm{RC} / \mathrm{CS}$, while the fresh weight of roots has significant positive correlation with ETo/CS. It is worth noting that $\mathrm{ETo} / \mathrm{CS}$ was correlated with all direct parameters, negatively with DR of leaves and roots and positively with fresh weight of leaves and roots, both in control and inoculated plants (Table 4).

Correlation coefficients calculated for the greenhouse experiment revealed significant negative relations of all the 
Table 4. Correlation coefficients between chlorophyll $a$ fluorescence (CF) parameters and disease rating (DR) and fresh weight of leaves and roots in plate assay

\begin{tabular}{lccccccccc}
\hline \multirow{2}{*}{$\begin{array}{l}\text { pF } \\
\text { parameters }\end{array}$} & Treatment & \multicolumn{2}{c}{ DR - leaf } & \multicolumn{2}{c}{ DR - root } & \multicolumn{2}{c}{ Fresh weight - leaf } & \multicolumn{2}{c}{ Fresh weight - root } \\
\cline { 3 - 9 } & & Inoculated & Control $^{1}$ & Inoculated & Control & Inoculated & Control & Inoculated & Control \\
\hline \multirow{2}{*}{$\mathrm{ABS} / \mathrm{CS}$} & Inoculated & $-0.230^{*}$ & - & $-0.353^{* *}$ & 0.176 & 0.126 & 0.107 & 0.133 & 0.187 \\
& Control & 0.198 & - & 0.031 & -0.025 & -0.140 & -0.175 & -0.049 & -0.110 \\
\multirow{2}{*}{$\mathrm{TR}_{\mathrm{O}} / \mathrm{CS}$} & Inoculated & $-0.297^{* *}$ & - & $-0.403^{* *}$ & 0.173 & 0.171 & 0.156 & 0.147 & $0.201^{*}$ \\
& Control & 0.149 & - & 0.005 & -0.028 & -0.168 & -0.170 & -0.059 & -0.119 \\
\multirow{2}{*}{$\mathrm{ET}_{\mathrm{O}} / \mathrm{CS}$} & Inoculated & $-0.391^{* *}$ & - & $-0.421^{* *}$ & $0.202^{*}$ & $0.346^{* *}$ & $0.245^{*}$ & $0.253^{*}$ & $0.246^{*}$ \\
& Control & 0.048 & - & -0.023 & 0.030 & -0.052 & 0.013 & 0.083 & 0.090 \\
\multirow{2}{*}{$\mathrm{DI}_{\mathrm{O}} / \mathrm{CS}$} & Inoculated & 0.029 & - & -0.109 & 0.134 & -0.037 & -0.062 & 0.055 & -0.092 \\
& Control & $0.236^{*}$ & - & 0.072 & -0.011 & -0.043 & -0.135 & -0.013 & -0.058 \\
$\mathrm{RC} / \mathrm{CSO}$ & Inoculated & $-0.374^{* *}$ & - & $-0.401^{* *}$ & 0.111 & $0.240^{*}$ & 0.180 & 0.195 & $0.212^{*}$ \\
& Control & 0.137 & - & 0.025 & -0.051 & -0.174 & -0.155 & -0.150 & -0.095 \\
$\mathrm{RC} / \mathrm{CS}$ & Inoculated & $-0.415^{* *}$ & - & $-0.407^{* *}$ & 0.082 & $0.257^{* *}$ & $0.213^{*}$ & 0.178 & 0.181 \\
& Control & -0.023 & - & -0.030 & -0.064 & -0.148 & -0.063 & -0.072 & -0.049 \\
\hline
\end{tabular}

$* P<0.05 ; * * P<0.01$

${ }^{1}-$ in control disease symptoms on leaves was only sporadically observed;

Table 5. Correlation coefficients between chlorophyll $a$ fluorescence (CF) parameters and disease rating (DR) and fresh weight of leaves revealed in greenhouse test

\begin{tabular}{llllll}
\hline \multirow{2}{*}{ CF parameters } & Treatment & \multicolumn{2}{c}{ DR - leaf } & \multicolumn{2}{c}{ Fresh weight - leaf } \\
\cline { 3 - 5 } & & Inoculated & Control & Inoculated & Control \\
\hline \multirow{2}{*}{$\mathrm{ABS} / \mathrm{CS}$} & Inoculated & $-0.576^{* *}$ & -0.186 & $0.655^{* *}$ & 0.077 \\
& Control & -0.159 & $-0.202^{*}$ & 0.048 & $0.508^{* *}$ \\
$\mathrm{TR}_{\mathrm{O}} / \mathrm{CS}$ & Inoculated & $-0.601^{* *}$ & -0.192 & $0.679^{* *}$ & 0.111 \\
& Control & -0.111 & -0.191 & 0.042 & $0.503^{* *}$ \\
$\mathrm{ET}_{\mathrm{O}} / \mathrm{CS}$ & Inoculated & $-0.623^{* *}$ & -0.139 & $0.655^{* *}$ & 0.115 \\
& Control & -0.002 & $-0.223^{*}$ & -0.068 & $0.518^{* *}$ \\
$\mathrm{DI}_{\mathrm{O}} / \mathrm{CS}$ & Inoculated & $-0.288^{* *}$ & -0.154 & $0.453^{* *}$ & 0.024 \\
& Control & $-0.289^{* *}$ & $-0.207^{*}$ & 0.065 & $0.429^{* *}$ \\
$\mathrm{RC} / \mathrm{CSO}$ & Inoculated & $-0.525^{* *}$ & -0.179 & $0.669^{* *}$ & 0.1359 \\
& Control & -0.171 & -0.094 & 0.135 & $0.552^{* *}$ \\
\hline
\end{tabular}

$* P<0.05 ; * * P<0.01$

studied CF parameters and DR of leaves for control and inoculated plants, but in infected plants the relation is much stronger (Table 5). When considering the fresh weight of leaves, the correlation coefficients are positive and significant both for control and infected plants.

Correlation coefficients between CF parameters and the direct traits observed in the field test (DR of head, number of kernel per head, weight of grains per head, kernel fractions (KD 1, KD2, KD3) showed that the most informative was DIo/CS (Table 6, Supplementary Table 4). This parameter had significant negative correlation with DR of head and the smallest grain fraction (KD1) and significant positive correlation with grain fraction $\mathrm{KD} 3$.

Data concerning the compatibility of CF parameters after inoculation assessed under various environments could provide useful information on whether plants express the same level of resistance in various conditions and various growth stages (Table 7). A significant positive correlation was revealed for a number of active reaction centers both oxidized and reduced ( $\mathrm{RC} / \mathrm{CSo}, \mathrm{RC} / \mathrm{CS}$, respectively) between 7-day seedlings in the plate test and 14-day seedlings in the greenhouse test. Additionally, significant positive correlation was observed for light energy absorption (ABS/ CS), amount of excitation energy trapped in PSII reaction 
Table 6. Correlation coefficients between chlorophyll $a$ fluorescence (CF) parameters and disease rating of heads (DR), number of grains per head (KNS), weight of grains per head (KWS) and kernel diameter: KD1, KD2 and KD3 revealed under field test

\begin{tabular}{|c|c|c|c|c|c|c|c|c|c|c|c|c|}
\hline \multirow{2}{*}{$\begin{array}{l}\mathrm{CF} \\
\text { parameter }\end{array}$} & \multirow{2}{*}{ Treatment } & DR & \multicolumn{2}{|c|}{ KNS } & \multicolumn{2}{|c|}{ KWS } & \multicolumn{2}{|c|}{ KD1 } & \multicolumn{2}{|c|}{ KD2 } & \multicolumn{2}{|c|}{ KD3 } \\
\hline & & $\begin{array}{l}\text { Inocu- } \\
\text { lated }\end{array}$ & $\begin{array}{c}\text { Inocu- } \\
\text { lated }\end{array}$ & Control & $\begin{array}{l}\text { Inocu- } \\
\text { lated }\end{array}$ & Control & $\begin{array}{c}\text { Inocu- } \\
\text { lated }\end{array}$ & Control & $\begin{array}{c}\text { Inocu- } \\
\text { lated }\end{array}$ & Control & $\begin{array}{c}\text { Inocu- } \\
\text { lated }\end{array}$ & Control \\
\hline \multirow{2}{*}{$\mathrm{ABS} / \mathrm{CS}$} & Inoculated & -0.167 & 0.021 & -0.100 & 0.208 & 0.106 & 0.173 & $0.204^{*}$ & -0.076 & -0.189 & -0.123 & -0.123 \\
\hline & Control & $-0.246^{*}$ & 0.144 & -0.041 & 0.184 & 0.034 & 0.131 & 0.091 & -0.037 & -0.065 & -0.106 & -0.102 \\
\hline \multirow{2}{*}{$\mathrm{TR}_{\mathrm{O}} / \mathrm{CS}$} & Inoculated & -0.062 & -0.014 & -0.079 & 0.165 & 0.131 & 0.099 & 0.182 & -0.086 & -0.187 & -0.046 & -0.065 \\
\hline & Control & $-0.223 *$ & 0.184 & -0.040 & $0.249 *$ & 0.133 & 0.047 & 0.005 & 0.068 & 0.030 & -0.086 & -0.091 \\
\hline \multirow{2}{*}{$\mathrm{ET}_{\mathrm{O}} / \mathrm{CS}$} & Inoculated & -0.014 & -0.078 & -0.062 & 0.124 & 0.183 & 0.073 & $0.207^{*}$ & -0.092 & $-0.212^{*}$ & -0.017 & -0.062 \\
\hline & Control & -0.121 & 0.178 & -0.047 & 0.233 & 0.153 & -0.084 & -0.047 & 0.108 & 0.056 & 0.019 & -0.004 \\
\hline \multirow{2}{*}{$\mathrm{DI}_{\mathrm{O}} / \mathrm{CS}$} & Inoculated & $-0.265^{* *}$ & 0.072 & -0.083 & 0.167 & -0.007 & $0.212 *$ & 0.119 & 0.001 & -0.071 & $-0.207^{*}$ & -0.169 \\
\hline & Control & -0.091 & 0.006 & -0.014 & -0.005 & -0.086 & 0.123 & 0.111 & -0.113 & -0.123 & -0.053 & -0.042 \\
\hline \multirow{2}{*}{ RC/CSo } & Inoculated & -0.040 & -0.063 & -0.083 & 0.163 & $0.203 *$ & 0.084 & $0.222^{*}$ & -0.139 & $-0.240^{*}$ & 0.002 & -0.048 \\
\hline & Control & -0.154 & 0.152 & -0.128 & $0.252 *$ & 0.093 & -0.010 & -0.050 & 0.071 & 0.053 & 0.057 & 0.013 \\
\hline \multirow{2}{*}{$\mathrm{RC} / \mathrm{CS}$} & Inoculated & 0.042 & -0.122 & -0.066 & 0.085 & 0.187 & 0.011 & 0.168 & -0.111 & -0.198 & 0.054 & 0.001 \\
\hline & Control & -0.085 & 0.170 & -0.033 & 0.196 & 0.141 & -0.126 & -0.060 & 0.107 & 0.059 & 0.059 & 0.028 \\
\hline
\end{tabular}

$* P<0.05 ; * * P<0.01$

$\mathrm{KD} 1, \mathrm{KD} 2$ and KD3 - kernel diameter: $>2.5 \mathrm{~mm} .2 .5-2.2 \mathrm{~mm}$ and $<2.2 \mathrm{~mm}$, respectively

Table 7. Correlation coefficients between chlorophyll $a$ fluorescence $(\mathrm{CF})$ parameters measured under plate assay, greenhouse and field tests

\begin{tabular}{|c|c|c|c|c|c|c|}
\hline \multirow{2}{*}{ Treatment } & \multicolumn{6}{|c|}{ CF parameters } \\
\hline & $\mathrm{ABS} / \mathrm{CS}$ & $\mathrm{TR}_{\mathrm{O}} / \mathrm{CS}$ & $\mathrm{ET}_{\mathrm{O}} / \mathrm{CS}$ & $\mathrm{DI}_{\mathrm{O}} / \mathrm{CS}$ & $\mathrm{RC} / \mathrm{CSo}$ & $\mathrm{RC} / \mathrm{CS}$ \\
\hline \multicolumn{7}{|c|}{ plate assay - greenhouse test } \\
\hline Inoculated & 0.099 & 0.119 & 0.179 & 0.0346 & $0.205^{*}$ & $0.222 *$ \\
\hline Control & -0.026 & -0.014 & -0.046 & -0.063 & 0.001 & -0.028 \\
\hline \multicolumn{7}{|c|}{ plate assay - field test } \\
\hline Inoculated & 0.167 & 0.151 & 0.028 & -0.091 & 0.121 & -0.029 \\
\hline control & -0.132 & 0.045 & -0.083 & -0.169 & 0.041 & 0.074 \\
\hline \multicolumn{7}{|c|}{ greenhouse test - field test } \\
\hline Inoculated & $0.222 *$ & $0.272 * *$ & 0.103 & -0.007 & $0.276^{* *}$ & 0.163 \\
\hline Control & -0.094 & -0.029 & 0.032 & -0.143 & 0.022 & 0.048 \\
\hline
\end{tabular}

$* P<0.05 ; * * P<0.01$

centers (TRo/CS) and number of oxidized active reaction centers ( $\mathrm{RC} / \mathrm{CSo})$ between 14-day seedlings grown in a greenhouse test and plants at field test.

\section{Discussion}

Fusarium culmorum along with Microdochium nivale and M. majus are major agents responsible for seedling blight and root rot as well as Fusarium head blight in barley and wheat. Seedling blight can significantly reduce seedling emergence and establishment as a result of infection, especially under high soil moisture favourable for fungi development, Fusarium head blight can result in notable yield reduction (Wang et al., 2006; Warzecha et al., 2011). Since publication of the first study to identify CF parameters from the JIP-test associated with fungal infection in wheat, this is the extension of the method proposed by Ajigboye et al. (2016), applied in barley $-F$. culmorm pathosystem. In winter wheat the changes in the PSII, as a response to fungal pathogen infection, were quantified from selected parameters resulting from the analysis of the fast fluorescence OJIP transient (Ajigboye et al., 2016). The authors proved that $\mathrm{CF}$ parameters were related significantly to known resistance ratings for foliar pathogen (Blmeria graminis) of wheat varieties, pathogen DNA of individual Oculimacula and Fusarium spp. generally existing in stem-base and ear 
diseases complexes.

The results of our studies showed a varied intensity of disease symptoms both in hulled and hull-less DH lines. Remarkably hulled lines revealed less root susceptibility to infection of $F$. culmorum expressed in DR and in fresh weight in the plate assay. The findings match results obtained by Warzecha et al. (2012) where less severe symptoms of the infection on leaves were found in hulled oat cultivars when compared to hull-less cultivars. Warzecha et al. (2012) reported that hull-less oat cultivars revealed a $20 \%$ higher reduction of leaf weight as compared with hulled cultivars. Similarly, in the case of the hulled genotypes the rating scale evaluation (no visual symptoms of the disease on seedling leaves) as well as seedling leaf weight values may suggest their greater resistance to $F$. culmorum.

As observed by Warzecha et al. (2015) in the set of barley DH lines the mean values of the seedling root weight were reduced more than twice (2.6 times) as much as the leaf weight, as with the evaluation in DR where the disease symptoms were three times more severe in roots in the group of hulled than in hull-less lines. Other authors showed that the destruction caused by Fusarium seedling blight is much greater in the root system (Grey and Mathre, 1988; Warzecha et al., 2012; Wojciechowski et al., 1997). Also, Ren et al. (2015) has postulated that the symptoms of FSB are predominantly restricted to the root and stem base of seedlings, unlike foliar diseases, since the disease is both seed- or soil-borne.

The root system damage has an impact on physiological processes: uptake and transport of water and mineral salts as well as assimilates distribution, which later affects the development of plants. The results obtained by Ren et al. (2015) indicated that Microdochium nivale and M. majus exhibit preferential pathogenicity towards certain plant tissues. It may also occur in the case of $F$. culmorum infection since severe damage was associated with roots.

In our studies inoculation in most cases decreased CF parameters, and the reduction was higher in the set of hullless lines comparing to hulled lines. This was most drastically observed in number of reduced active reaction centers (RC/CS) under greenhouse conditions. Under field conditions the most affected by infection parameter was light energy absorption by leaf cross-section (ABS/CS), whereas $\mathrm{ETo} / \mathrm{CS}$ and RC/CS did not changed significantly. In the present paper the analysis of variance revealed that treatment, genotype, and the interaction of both factors were significant for almost all measured CF parameters (apart from DIo/CS, where the treatment was not significant) in plate assay. Therefore, it was observed that in the population of homozygous $\mathrm{DH}$ lines obtained from the $\mathrm{F}_{1}$ genera- tion of two parents differing in their resistance/susceptibility to $F$. culmorum infection.

Berger et al. (2007) reported reduced CF parameters (Fv/ Fm) significantly associated with biotic stress. Also, Ren et al. (2015) reported that infection caused by M. majus significantly reduced the maximum efficiency of PSII in the set of studied wheat cultivars. In our studies this tendency was most visible in the reduced number of active reaction centers in hull-less lines, while in the set of hulled lines this reduction was insignificant. In the field test infection caused not only a reduction of CF parameters, but also yield-related traits. Ajigboye et al. (2016) also observed a significant reduction in PSII efficiency after wheat plants were infected with severe pathogens like $F$. avenaceum and $F$. culmorum. The above authors observed a significant decrease of $\mathrm{Fv} / \mathrm{Fm}$ and active reaction centers on one hand, but an increase of total dissipation of excess excitation energy from PSII reaction centers on the other. Moreover, infection also caused a decrease in electron transport, as was observed by Pinto et al. (2000), who postulated that impairment of photosynthetic efficiency was predominantly caused by the decrease of the mentioned CF parameter.

It is noticeable that the reduction in the number of active reaction centers was greater in hull-less DH lines, while in the set of hulled lines the changes were considerably lower. The above information confirms the observations of other authors that the redox state of PSII can regulate many processes, including the expression of photosynthetic genes and chloroplast biogenesis (Pfannschmidt et al., 2001), leaf colouring during the senescence and anthocyanin biosynthesis in cold (Feild et al., 2001), and also growth rate by changes in hormonal balance (ABA/GAs) (Rapacz, 1998; Rapacz et al., 2003). Based on our results, it can be stated that the redox state of PSII can play an important role in plants' defense mechanisms. A similar tendency in CF parameters $(\mathrm{ABS} / \mathrm{CS}, \mathrm{DIo} / \mathrm{CS}$ and $\mathrm{RC} / \mathrm{CS}$ were significant, whereas $\mathrm{TRo} / \mathrm{CS}, \mathrm{ETo} / \mathrm{CS}$ and $\mathrm{RC} / \mathrm{CSo}$ were insignificant) was observed in our experiments in plate assay, greenhouse test and field experiment.

If we consider stress caused by infection, the formation of the CF parameter concerning amount of energy dissipated from PSII, estimated as (ABS/CS - TRo/CS) - (DIo/ $\mathrm{CS}$ ), could be interesting. In plate assay this parameter was insignificant but in greenhouse test and field experiment it was significant. In the set of infected hulled lines the amount of energy dissipated from PSII was much higher than in the set of hull-less lines. This could indicate that plants under stress caused by infection can fight with some damage of PSII by higher energy dissipation as suggested by Huner et al. (1993). 
In our study inoculation significantly influenced the following CF parameters: $\mathrm{ABS} / \mathrm{CS}, \mathrm{TRo} / \mathrm{CS}, \mathrm{ETo} / \mathrm{CS}$, $\mathrm{RC} / \mathrm{CS}$, $\mathrm{RC} / \mathrm{CS}$ in plate assay. All CF parameters were significantly influenced by treatment in greenhouse test and three $\mathrm{CF}$ parameters: $\mathrm{ABS} / \mathrm{CS}$, DIo/CS, $\mathrm{RC} / \mathrm{CS}$ in field test. Similar results were reported by Warzecha et al. (2015) who demonstrated highly significant differences between the DH lines and the interaction for the maximum photochemical efficiency (Fv/Fm), and the overall performance index of PSII (PI). Hull-less lines had lower mean PI values than hulled lines for inoculated plants compared to the control plants. Ajigboye et al. (2016), suggest that specific CF parameters that enable the fast detection and differentiation of plant responses may be related to trophic relationship. Necrotrophic pathogens must kill the host tissue to get nutrients (Perfect and Green, 2001). The above authors postulated that wheat response to necrotrophic pathogens like $F$. avenauceum and $F$. culmorum were quantified more closely with CF parameters related to PSII efficiency and active PSII RC possibly because of rapid damage associated with aggressive infection. The reduction of photosynthesis efficiency caused by necrotrophic pathogens as presented in previous studies was directly associated with decreasing chlorophyll content in necrotic tissue and decreasing photosynthetic efficiency in green foliar tissue caused by decomposition of cell components, and eliciting enzymes, toxins, and reactive oxygen species from pathogens in order to colonize host tissue (Mengiste, 2012; Warzecha et al., 2015).

In plate assay CF parameters CF parameters had a significant negative correlation with DR (disease rating) of leaves and roots. Higher values of DR (more severe damage of roots and leaves) resulted in lower performance of PSII. So, it provides an extra opportunity to access the level of resistance of various genotypes via an indirect test. In greenhouse, higher values of infected plants FW are connected with better efficiency of PSII. Each of the studied CF parameters could be informative regarding the level of resistance of genotypes.

The impact of infection evaluated under field conditions was clearly visible looking at direct parameters: DR of heads, number of grains per head, weight of grains per head and grain fractions. The reduction of grain size could be associated with the disease impact (or the result of infection enhancing small grain as a disturbance of grain development, storage material shortage and unsettled assimilate transportation). Considering CF parameters the most informative was DIo/CS, which had a significant negative correlation with DR of head and the smallest grain fraction $\mathrm{KD} 1(<2.2 \mathrm{~mm})$ and a significant positive correlation with grain fraction KD3 (>2.5 mm). Therefore, higher values of $\mathrm{DIo} / \mathrm{CS}$ were related to weaker scab symptoms, as revealed by visual assessment of heads and decreased amount of smallest grain fraction.

The CF parameters were also examined according to their compatibility among the three environments (plate essay for resistance, greenhouse test, field test). It was revealed that more parameters had significant positive correlation in the greenhouse test and field test - ABS/CS, TRo/ $\mathrm{CS}$, and RC/CS. Only two parameters (RC/CSo and RC/ $\mathrm{CS}$ ) had a significant positive correlation in the plate assay for resistance and greenhouse test.

Both plate assay and greenhouse test assessed susceptibility to FSB. However, the method of inoculation was different. Therefore, the more relevant method based on CF parameters, which better explains the linkage between FSB and FHB, is inoculating the grain and sowing them in the soil (greenhouse test). The differences in susceptibility assessment to $M$. majus with various methods of pathogen application was reported by Ren et al. (2015), in which the CF parameters better correlated with FSB or plant trait assessment when comparing the detached leaf inoculation method to soil inoculation.

\section{Conclusions}

After infection of barley DH lines by $F$. culmorum most CF parameters were significantly reduced, and the reduction was higher in the set of hull-less lines compared to hulled lines. The plate assay revealed that the amount of energy used for electron transport (ETo/CS) is negatively correlated with disease rating of leaves and roots and positively with fresh weight of leaves and roots. It provides an extra opportunity to assess the level of resistance of various genotypes via an indirect test.

In the greenhouse test, higher fresh weight of infected plants is connected with better efficiency of PSII based on chlorophyll fluorescence parameters. Each of the studied CF parameters could reveal the resistance levels of genotypes. The field test revealed that the higher DIo/CS values are related to weaker scab symptoms, as revealed by visual assessment of heads and a decreased amount of smallest grain fraction associated with the disease impact. FSB assessed under greenhouse conditions better correlates with FHB under field conditions than plate assay for resistance when considering CF parameters.

Two lines were distinguished according to their performance in laboratory tests, R63N/9 and R63N/4, which possessed the lowest DR, the highest number of grains per ear and the highest values of CF parameters. The lines men- 
tioned above were also least affected by Fusarium head blight.

\section{References}

Adamski, T., Chełkowski, J., Goliński, P., Kaczmarek, Z., Kostecki M., Perkowski, J., Surma, M. and Wiśniewska, H. 1999. Yield reduction and mycotoxin accumulation in barley doubled haploids inoculated with Fusarium culmorum (W.G.Sm.) Sacc. J. Appl. Genet. 40:73-84.

Ajigboye, O. O., Murchie, E. H. and Ray, R. V. 2014. Foliar application of isopyrazam and epoxiconazole improves photosystem II efficiency, biomass and yield in winter wheat. Pestic. Biochem. Physiol. 114:52-60.

Ajigboye, O. O., Bousquet L., Murchie E. H. and Ray R. V. 2016. Chlorophyll fluorescence parameters allow the rapid detection and differentiation of plant responses in three different wheat pathosystems. Funct. Plant Biol. 43:356-369.

Arseniuk, E., Góral, T. and Czembor, H. J. 1993. Reaction of triticale, wheat and rye accessions to graminaceous Fusarium spp. infection at the seedling and adult plant growth stages. Euphytica 70:175-183.

Arseniuk, E., Foremska, E. and Góral, T. and Chełkowski, J. 1999. Fusarium head blight reactions and accumulation of deoxynivalenol (DON) and some of its derivatives in kernels of wheat. triticale and rye. J. Phytopathol. 147:577-590.

Baker, N. R. and Rosenqvist, E. 2004. Applications of chlorophyll fluorescence can improve crop production strategies: an examination of future possibilities. J. Exp. Bot. 5:1607-1621.

Bauriegel, E., Giebel, A. and Herppich, W. B. 2010. Rapid Fusarium head blight detection on winter wheat ears using chlorophyll fluorescence imaging. J. Appl. Bot. Food Qual. 83:196-203.

Bolhar-Nordenkampf, H. R. and Öquist, G. 1993. Chlorophyll fluorescence as a tool in photosynthesis research. In: Photosynthesis and production in a changing environment: a field and laboratory manual, eds. by D. O. Hall, J. M. O. Scurlock, H. R. Bolhàr-Nordenkampf, R. C. Leegood and S. P. Long, pp. 193-206. Springer, Dordrecht, The Netherlands.

Bottalico, A. and Perrone, G. 2002. Toxigenic Fusarium species and mycotoxins associated with head blight in small - grain cereals in Europe. Eur. J. Plant Pathol. 108:611-624.

Buerstmayr, H., Ban, T. and Anderson, J. A. 2009. QTL mapping and marker-assisted selection for Fusarium head blight resistance in wheat: a review. Plant Breed. 128:1-26.

Chełkowski, J. and Mańka, M. 1983. The ability of Fusaria pathogenic to wheat, barley and corn to produce zearalenone. J. Phytopathol. 106:354-359.

Chełkowski, J., Kaptur, P., Tomkowiak, M., Kostecki M., Goliński P., Ponitka, A., Ślusarkiewicz-Jarzina, A. and Bocianowski, J. 2000. Moniliformin accumulation in kernels of triticale accessions inoculated with Fusarium avenaceum in Poland. $J$. Phytopathol. 148:433-449.

Cowger, C., Patton-Ozkurt, J., Brown-Guedira, G. and Perugini, L.
2009. Post-anthesis moisture increased Fusarium head blight and deoxynivalenol levels in North Carolina winter wheat. Phytopathology 99:320-327.

Czyczyło-Mysza, I., Tyrka, M., Marcińska, I., Skrzypek, E., Karbarz, M., Dziurka, M., Hura, T., Dziurka, K. and Quarrie, S. A. 2013. Quantitative trait loci for leaf chlorophyll fluorescence parameters. chlorophyll and carotenoid contents in relation to biomass and yield in bread wheat and their chromosome deletion bin assignments. Mol. Breed. 32:189-210.

Demetriou, G., Neonaki, C., Navakoudis, E. and Kotzabasis, K. 2007. Salt stress impact on the molecular structure and function of the photosynthetic apparatus: the protective role of polyamines. Biochim. Biophys. Acta 1767:272-280.

Desjardins, A. E. 2006. Fusarium mycotoxins. Chemistry, genetics. and biology. APS Press, St. Paul, MN, USA. 260 pp.

Foroud, N. A. and Eudes, F. 2009. Trichothecenes in cereal grains. Int. J. Mol. Sci. 10:147-173.

Fracheboud, Y. and Leipner, J. 2003. The application of chlorophyll fluorescence to study light, temperature and drought stress. In: Practical applications of chlorophyll fluorescence in plant biology, eds. by J. R. DeEll and P. M. A. Tiovonen, pp. 125-150. Springer, Boston, MA, USA.

Gorbe, E. and Calatayud, A. 2012. Applications of chlorophyll fluorescence imaging technique in horticultural research: a review. Sci. Hortic. 138:24-35.

Grey, W. and Mathre, D. E. 1988. Evaluation of spring barley for reaction to Fusarium seedling blight and root rot. Can. J. Plant Sci. 68:23-30.

Huner, N. P., Öquist, G., Hurry, V. M., Krol, M., Falk, S. and Griffith, M. 1993. Photosynthesis, photoinhibition and low temperature acclimation in cold tolerant plants. Photosynth. Res. 37:19-39.

Imathiu, S. M., Hare, M. C., Ray, R. V., Back, M. and Edwards, S. G. 2010. Evaluation of pathogenicity and aggressiveness of $F$. langsethiae on oat and wheat seedlings relative to known seedling blight pathogens. Eur. J. Plant Pathol. 126:203-216.

Inch, S. A. and Gilbert, J. 2003. Survival of Gibberella zeae in Fusarium-Damaged wheat kernels. Plant Dis. 83:282-287.

Kasha, K. J. and Kao, K. N. 1970. High frequency haploid production in barley (Hordeum vulgare L.). Nature 225:874-876.

Ma, H. X., Ge, H. J., Zhang, X., Lu, W. Z., Yu, D. Z., Chen, H. and Chen, J. M. 2009. Resistance to Fusarium head blight and deoxynivalenol accumulation in Chinese barley. J. Phytopathol. 157:166-171.

Magan, N., Hope, R., Colleate, A. and Baxter, E. S. 2002. Relationship between growth and mycotoxin production by Fusarium species. biocides and environment. Eur. J. Plant Pathol. 108:685-690.

Mardi, M., Pazouki, L., Delavar, H., Kazemi, M. B., Ghareyazie, B., Steiner, B., Nolz, R., Lemmens, M. and Buerstmayr, H. 2006. QTL analysis of resistance to Fusarium head blight in wheat using a 'Frontana'- derived population. Plant Breed. 125:313-317.

Marin, S., Ramos, A. J., Cano-Sancho, G. and Sanchis, V. 2013. 
Mycotoxins: occurrence, toxicology. and exposure assessment. Food Chem. Toxicol. 60:218-237.

Maxwell, K. and Johnson, G. N. 2000. Chlorophyll fluorescence - a practical guide. J. Exp. Bot. 51:659-668.

Mengiste, T. 2012. Plant immunity to necrotrophs. Annu. Rev. Phytopathol. 50:267-294.

Mesterházy, A. 1995. Types and components of resistance to Fusarium head blight of wheat. Plant Breed. 114:377-386.

Mesterházy, A. 2002. Role of deoxynivalenol in aggressiveness of Fusarium graminearum and F. culmorum and in resistance to Fusarium head blight. Eur. J. Plant Pathol. 108:675-684.

Mesterházy, A., Bartók, T., Mirocha, C. G. and Komoróczy, R. 1999. Nature of wheat resistance to Fusarium head blight and the role of deoxynivalenol for breeding. Plant Breed. 118:97110.

Miedaner, T. 1997. Breeding wheat and rye for resistance to Fusarium diseases. Plant Breed. 116:201-220.

Miedaner, T., Reinbrecht, C., Lauber, U., Schollenberger, M. and Geiger, H. H. 2001. Effects of genotype and genotype x environment interaction on deoxynivalenol accumulation and resistance to Fusarium head blight in rye, triticale, and wheat. Plant Breed. 120:97-105.

Nielsen, L. K., Jensen, J. D., Nielson, G. C., Spliid, N. H., Thomsen, I. K., Justesen, A. F., Collinge, D. B. and Jørgensen, L. N. 2011. Fusarium head blight of cereals in Denmark: species complex and related mycotoxins. Phytopathology 101:960969.

Nielsen, L. K., Justesen, A. F., Jensen, J. D. and Jørgensen, L. N. 2013. Microdochium nivale and Microdochium majus in seed samples of Danish small grain cereals. Crop Prot. 43:192200.

Nielsen, L. K., Cook, D. J., Edwards, S. G. and Ray, R. V. 2014. The prevalence and impact of Fusarium head blight pathogens and mycotoxins on malting barley quality in UK. Int. J. Food Microbiol. 179:38-49.

O’Neill, P. M., Shanahan, J. F. and Schepers, J. S. 2006. Use of chlorophyll fluorescence assessments to differentiate corn hybrid response to variable water conditions. Crop Sci. 46:681687.

Pereira, W. E., de Siqueira, D. L., Martínez, C. A. and Puiatti, M. 2000. Gas exchange and chlorophyll fluorescence in four citrus rootstocks under aluminium stress. J. Plant Physiol. 157:513-520.

Perfect, S. E. and Green, J. R. 2001. Infection structures of biotrophic and hemibiotrophic fungal plant pathogens. Mol. Plant Pathol. 2:101-108.

Pfannschmidt, T., Allen, J. F. and Oelmüller, R. 2001. Principles of redox control in photosynthesis gene expression. Physiol. Plant. 112:1-9.

Pickering, R. A. and Devaux, P. 1992. Haploid production: Approaches and use in plant breeding. In: Barley: Genetics. Biochemistry, molecular biology and biotechnology, ed. by P. R. Shewry, pp. 519-547. CAB International, Wallingford, UK.

Pinto, L. S. R. C., Azevedo, J. L., Pereira, J. O., Vieira, M. L. C. and Labate, C. A. 2000. Symptomless infection of banana and maize by endophytic fungi impairs photosynthetic efficiency. New Phytol. 147:609-615.

Rapacz, M. 1998. The after-effects of temperature and irradiance during early growth of winter oilseed rape (Brassica napus L. var. oleifera cv. Gorczanski) seedlings on the progress of their cold acclimation. Acta Physiol. Plant. 20:73-78.

Rapacz, M., Waligórski, P. and Janowiak, F. 2003. ABA and gibberellin- like substances during prehardening, cold acclimation, de- and reacclimation of oilseed rape. Acta Physiol. Plant. 25:151-161.

Ren, R., Yang X. and Ray, R. V. 2015. Comparative aggressiveness of Microdochium nivale and M. majus and evaluation of screening methods for Fusarium seedling blight resistance in wheat cultivars. Eur. J. Plant Pathol. 141:281-294.

Rolfe, S. A. and Scholes, J. D. 2010. Chlorophyll fluorescence imaging of plant pathogen interactions. Protoplasma 247:163-175.

Schroeder, H. W. and Christiansen, J. J. 1963. Factors affecting resistance of wheat to scab caused by Gibberella zeae. Phytopathology 53:831-838.

Smillie, R. M. and Nott, R. 1982. Salt tolerance in crop plants monitored by chlorophyll fluorescence in vivo. Plant Physiol. 70:1049-1054.

Snijders, C. H. 2004. Resistance in wheat to Fusarium infection and trichothecene formation. Toxicol. Lett. 153:37-46.

Strasser, B. J. and Strasser, R. J. 1995. Measuring fast fluorescence transients to address environmental questions: the JIP-Test. In: Photosynthesis: from light to biosphere, ed. by P. Mathis, pp. 977-980. KAP Press, Dordrecht, The Netherlands.

Strasser, R. J. and Tsimilli-Michael, M. 1998. Activity and heterogeneity of PS II probed in vivo by the chlorophyll-a fluorescence rise O-(K)-J-I-P. In: Photosynthesis: mechanisms and effects, ed. by G. Garab, pp. 4321-4324. KAP Press, Dordrecht, The Netherlands.

Strasser, R. J., Srivastava, A. and Tsimilli-Michael, M. 2000. The fluorescence transient as a tool to characterize and screen photosynthetic samples. In: Probing photosynthesis: mechanisms, regulation and adaptation, eds. by $\mathrm{M}$. Yunus, $\mathrm{U}$. Pathre and $\mathrm{P}$. Mohanty, pp. 445-483. Taylor and Francis, London, UK.

Wang, H., Hwang, S. F., Eudes, F., Chang, K. F., Howard, R. J. and Turnbull, G. D. 2006. Trichothecenes and aggressiveness of Fusarium graminearum causing seedling blight and root rot in cereals. Plant Pathol. 55:224-230.

Warzecha, T., Adamski, T., Kaczmarek, Z., Surma, M., Goliński, P., Perkowski, J., Chełkowski, J., Wiśniewska, H., Krystkowiak, K. and Kuczyńska, A. 2010. Susceptibility of hulled and hulless barley doubled haploids to Fusarium head blight. Cereal Res. Commun. 38:220-232.

Warzecha, T., Adamski, T., Kaczmarek Z., Surma, M., Chełkowski, J., Wiśniewska, H., Krystkowiak, K. and Kuczyńska, A. 2011. Genotype-by-Environment interaction of barley DH lines infected with Fusarium culmorum (W.G.Sm.) Sacc. Field Crops Res. 120:21-30. 
Warzecha, T., Zieliński, A., Skrzypek, E., Wójtowicz, T. and Moś, M. 2012. Effect of mechanical damage on vigor. physiological parameters. and susceptibility of oat (Avena sativa) to Fusarium culmorum infection. Phytoparasitica 40:29-36.

Warzecha, T., Skrzypek, E. and Sutkowska, A. 2015. Effect of Fusarium culmorum infection on the selected physiological and biochemical parameters of barley (Hordeum vulgare L.) DH lines. Physiol. Mol. Plant Pathol. 89:62-69.

Wiśniewska, H., Stępień, Ł., Waśkiewicz, A., Beszterda, M., Góral, T. and Belter, J. 2014. Toxigenic Fusarium species infecting wheat heads in 2009 in selected regions of Poland. Central Eur. J. Biol. 9:163-172.
Wojciechowski, S., Chełkowski, J., Ponitka, A. and ŚlusarkiewiczJarzina, A. 1997. Evaluation of spring and winter wheat reaction to Fusarium culmorum and Fusarium avenaceum. J. Phytophatol. 145:99-103.

Yang, Z. P., Gilbert, J., Fedak, G. and Somers, D. J. 2005. Genetic characterization of QTL associated with resistance to Fusarium head blight in a doubled-haploid spring wheat population. Genome 48:187-196.

Živcák, M., Brestic, M., Olšovská, K. and Slamka, P. 2008. Performance index as a sensitive indicator of water stress in Triticum aestivum L. Plant Soil Environ. 54:133-139. 